\title{
Evaluation Mechanism for Adaptive Education
}

\author{
Sonal Chawla
}

\begin{abstract}
Currently, much of the courseware and resources in Adaptive Education Hypermedia are unstructured and isolated from each other thereby lacking in quality too. Therefore, this research aims to provide a research framework to develop a system for the learning process which is able to store the quality content and depending on cognitive state of the student, preset a qualitative and quantitative result of their learning level. Thus, the objective of this research paper is threefolds. It firstly looks at the available literature related to tools techniques for Learning Object(LO) Evaluation and metrics Secondly, it proposes a framework for the design and dissemination of Adaptive education combining learning theory with LO evaluation systems. The paper finally concludes by identifying a relationship between the determined learning style profile, the assigned task and the chosen representation of the content.
\end{abstract}

Index Terms-Learning objects, metadata, evaluation metrics.

\section{INTRODUCTION}

Adaptive Education Hypermedia (AEH) is a challenging research area that helps to improve the learning of the students adjusting the content and navigation alternatives to their characteristics. It is a process where learners gain knowledge and skills interacting with learning resources, activities and other students. Learning design [1] details this process, considering learning goals, prerequisites and expected outcomes to indicate learning activities, sequencing and learning materials.

Advanced techniques are already being used in higher education to facilitate learning and teaching, but inadequacies still exist [2]. Currently, much of the courseware and resources are unstructured and isolated from each other [3]. The genesis of adaptive learning systems is from the artificial intelligence (AI) research. In the early 1980 there was significant development of systems to provide intelligent response to users interacting with the computers. The early AI research developed into three overlapping streams, namely, knowledge based expert systems, neural networks and genetic algorithms. These technologies were used primarily in adaptive control systems that managed the difficult task of controlling electromechanical actuators to adapt to the given situation and respond accordingly. The artificial intelligence systems were based on strategies to learn user's behavior and respond accordingly. The conceptual and philosophical differences of these approaches led to the learning systems that were either influenced by the connectionists model that created supervised neural nets or unsupervised self organizing

Manuscript received March 25, 2013; revised May 17, 2013.

Sonal Chawla is with the Department of Computer Science and Applications, Panjab University, India. (E-mail: sonal_chawla@yahoo.com) maps or reduction of the knowledge domain into set-of symbolic representations leading to knowledge based expert rules that can be fired to resolve a decision for the given situation. Unfortunately, the learning management systems (LMS), learning content management systems (LCMS) and even the course management systems (CMS) completely have been devoid of any tool that allowed intelligent tutoring system to become part of the learning system to help individual learners to learn. This mainly depends on the quality of content being presented to the learner[4]-[6]

However, the evaluation of content is of extreme importance to maintain quality. Evaluation is essential for every aspect of designing LOs, including

- Identifying learners and their needs

- Conceptualizing a design

- Developing prototypes

- Implementing and delivering instruction

- Improving the evaluation itself.

Most searches in LO repositories return vast materials without any assurance of value and quality, making it difficult for users to decide which of them best suits their requirements. There is, in fact, a need for an automatic assessment system that provides the users highly rated instructional material.

\section{LITERATURE REVIEW}

There were two approaches followed for evaluation of LOs

- Summative Approach deals with final evaluation of learning objects based on informal interviews or surveys, frequency of use and learning outcome.

- Formative Approach works during the development phase of learning objects where feedback is solicited from small groups at regular intervals.

Presently the approaches followed for evaluation of LOs are in the form of:

\section{- LO Evaluation Tools/ Techniques}

- LO Evaluation Metrics

Tools/ Techniques currently being used by various repositories for evaluation of LOs are Convergent Evaluation Model, LORI, Peer Review, LOEM: Multi-Component Model for Learning Object Evaluation Metrics, LOES, HEODAR, MECOA etc.

LORI[6], [7] is used to evaluate the quality of learning objects with respect to nine dimensions using a five point rating scale ranging from low to high:

Content Quality: includes Veracity, accuracy, balanced presentation of ideas, and appropriate level of detail

Learning Goal Alignment: includes Alignment among learning goals, activities, assessments, and learner characteristics

Feedback and Adaptation: includes Adaptive content or feedback driven by differential learner input or learner 
modelling

Motivation: includes Ability to motivate, and stimulate the interest or curiosity of, an identified population of learners.

Presentation Design: includes Design of visual and auditory information for enhanced learning and efficient mental processing

Interaction: includes Usability Ease of navigation, predictability of the user interface, and the quality of UI help features

Accessibility: includes Support for learners with disabilities

Reusability: includes Ability to port between different courses or learning contexts without modification

Standards Compliance: includes Adherence to international standards and specification

The Convergent Participation Model [6]-[8] has two cycles. The first cycle evaluates the LO asynchronously and the second cycle gets the integrated view

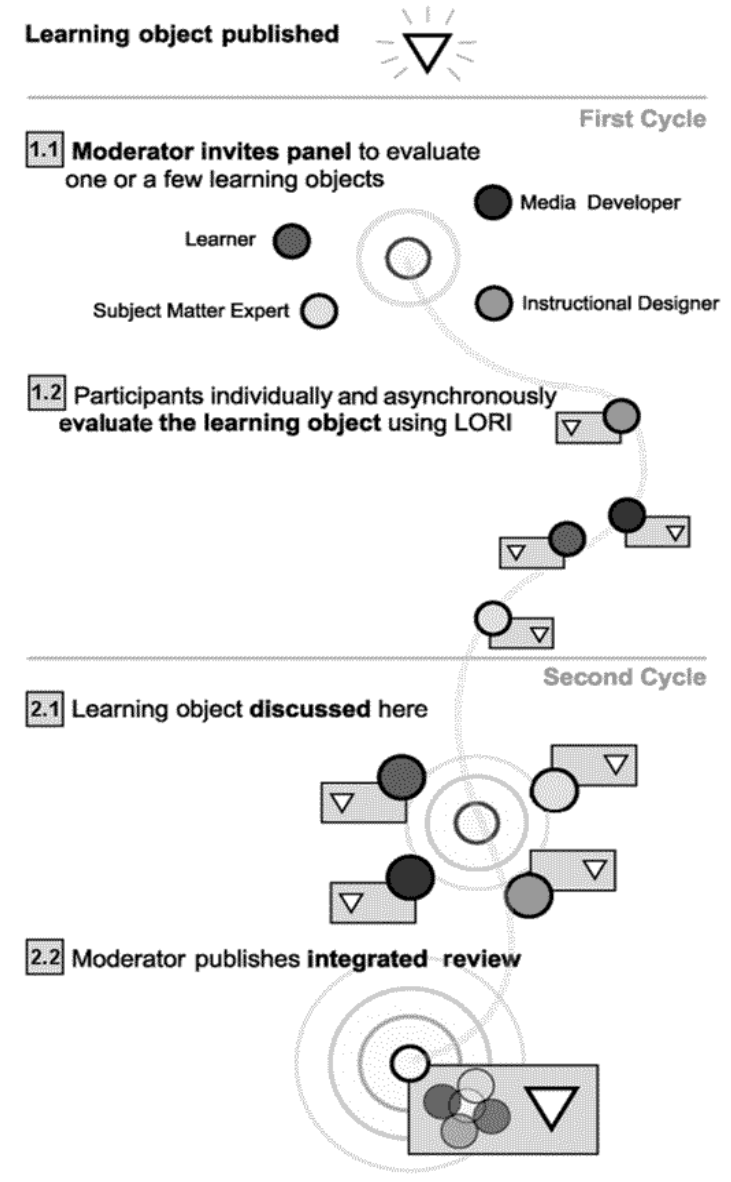

Fig. 1. Convergent participation model

Peer Reviews evaluate three dimensions of LO i.e. Quality of content, potential effectiveness as a teaching - learning tool and Ease of use. Each aspect is rated on a scale from 1 to 5, rating objects from "poor" to "excellent". The weighted mean of the three dimensions will be the final value of the learning object evaluation

Kay \& Knaack [9] proposed a multi component model LOEM which focused on five main criteria: interactivity, design, engagement, usability, content etc.

Munoz \& Conde [10] designed and developed a model HEODAR that automatically evaluates the LOs and produces a set of information that can be used to improve those LOs.
The tool is implemented in the University of Salamanca framework and initially integrated with LMS called Moodle but the results are not yet tested.

Eguigure \& Zapata [10] proposed a model for Quality Evaluation of Learning Objects (MECOA) which defines six indicators Content, Performance, Competition, self-management, Meaning, Creativity

These indicators are evaluated by four actors: teachers, student, experts and pedagogues and implemented in AGORA platform.

\section{LimitATIONS OF THE CURRENT RESEARCH WORK}

The Current Research work highlighted above evaluates qualitative aspect of learning objects. It depends extensively on human review which is costly and time consuming. Also studies have been conducted to evaluate limited no. of objects. [11]-[13]

Different repositories [14], [15] have different evaluation systems, thus making it difficult to sort the results returned for several repositories. Since metrics is the measurement of a particular characteristic. Researchers have proposed various metrics for quantitative analysis of different dimensions of learning objects such as

Quality Metrics [16]-[19]: small calculation performed over the values of different fields of the metadata record in order to gain insight of various quality characteristics such as completeness, accuracy, provenance, conformance to expectations, coherence and consistency, timeliness and accessibility.

Reusability Metrics [20], [21]: Reusability is the degree to which a learning object can work efficiently for different users in different digital environments and in different educational contexts over time. The factors which are required to be considered are modifiability, granularity, traceability, platform independence etc.

Relevance Ranking Metrics: ranks the LOs on the basis of information available from usage and the context of learning for efficient retrieval.

Cost Metrics: measures the cost of LOs reuse as a process rather than measuring only the potential reusability of individual LOs.

Learning Style Metrics: evaluates the compatibility of LO with different learning styles so as to ensure that the learning material will provide an equal learning experience for student with different pedagogical requirements.

\section{RESEARCH OBJECTIVES}

The research therefore attempts to answer the following questions

- How should the metrics and models evaluating individual parameters of LOs be applied on large and federated LORs?

- How the metrics evaluating different parameters of LO be combined to give overall rating about the quality of LOs?

- How should the metrics calculating various parameters of LOs be adapted to environment in which only the top-k objects of each repository are known? 
- How should the ranking made by different LORs be aggregated?

\section{Methodology}

The methodology used for the present study is to develop an agent based system that evaluates the LO in terms of defined parameters such as quality of metadata, reusability, granularity and ranking and gives assurance regarding quality and value of the object to the whole community.

The Research Methodology was divided into two phases:

- Phase I: Analysis Phase.

- Phase II: Design Phase.

Phase I involved the detailed study of models, theories, frameworks and algorithms proposed by researchers for LO evaluation.

Phase II provided the detailed Design of the system as shown below

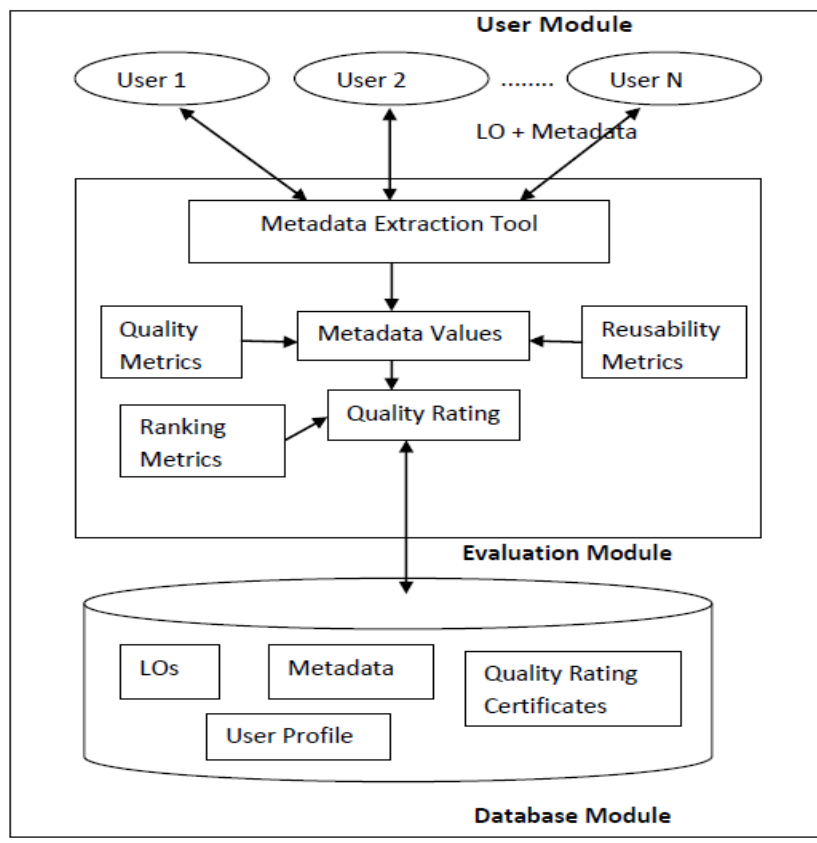

Fig. 2. Detailed design of the evaluation mechanism for adaptivity

Here the system was divided into three modules: the User Module, Evaluation Module and the Database Module. The User Module provided the interface as a front end.The Evaluation module had various dimensions to it. It combined the properties of Quality Metrics[22]-[24], Reusability metrics and Ranking Metrics thereby allocating quality rating to the Learning Object .The Database module stored the Learning Objects as a repository and their metadata values for extraction. Each Learning Object that became a part of this database had the quality rating from the evaluation module. Along with this the user profile was stored that was matched to provide the learning object suitable to the learning style of the individual. This adaptive mechanism involved the use of artificial Intelligence and Neural Network techniques. The framework, thus, provided the Quality LOs to be a part of the repository and also these LOs could be extracted based on their metadata tags and their quality indicators suiting the learning styles of the learners whose profile too were a part of the database.

\section{Significance of the study:}

Ratings and quantitative assessments aid individual users in searching and selecting reliable objects. Evaluations provide guidance on how best to use an object. Quality was increased by evaluating the object before submitting it to any repository. Evaluation standards drove the practices of designers and developers. Participation in evaluation activities contributed to the professional development of those who work with LOs. Positive evaluations promoted social recognition of skilled designers and developers. A trusted evaluation system was an essential step toward the development of a workable business model for the economic exchange of LOs.

\section{CONCLUSIONS}

Review of literature shows that research on the evaluation of LOs in terms of quantitative factors is very limited, partially because of abundance of qualitative tools and dependency on expert review. The above study unveils the requirement of a quantitative automatic assessment system for evaluation of LO in terms of defined parameters so as to get assurance about the quality of LOs because of large dissemination of LOs. Expert system presently evaluates LOs efficiently but due to limited number of experts, difference in opinion and presence of biasness, this system does not work efficiently and covers limited number of objects. The proposed system acts as a certification mechanism for the quality of LOs which gives reasonable assurance to LO users about quality and content. The adaptive mechanism involving the presentation of the LO suiting the learning style of the learners is a major step towards adaptivity.

\section{REFERENCES}

[1] Learning Technology Standards Committee, Draft Standard for Learning Object Metadata, IEEE Standard 1484.12.1, New York: Institute of Electrical and Electronics Engineers, pp. 45, 2002

[2] D. A. Wiley. Connecting learning objects to instructional theory: A definition, a metaphor, and taxonomy. [Online]. Avaialble: http://reusability.org/read/chapters/wiley.doc

[3] B. Collis and A. Strijker. Technology and human issues in reusing learning objects. Journal of Interactive Media in Education. [Online]. Available: http://jime.open.ac.uk/2004/4/collis-2004-4.pdf. 2004

[4] M. Haughey and B. Muirhead, "Evaluating learning objects for schools," E-Journal of Instructional Science and Technology, vol. 8, no. 1,2005

[5] Kay and Knaack, "A multi-component model for accessing learning objects: the learning object evaluation metrics (LOEM)" Australasian Journal of Educational Technology, vol. 24, no. 5, 2008, pp. 574-591.

[6] Nesbit and Belfer, "A convergent participation model for evaluation of learning objects," Canadian Journal of Learning \& Technology, vol. 28 , no. 3, 2002, pp. 105-200.

[7] J. Nesbit and K. Belfer, "Collaborative evaluation of learning objects," in Online Education Using Learning Objects, R. McGreal (ed.), New York: RoutledgeFalmer, 2004, pp. 138-153.

[8] Kay and Knaack, "Developing learning objects for secondary school students: a multi-component model," Interdisciplinary Journal of Knowledge and Learning Objects, vol. 1, 2005, pp. 229-254.

[9] K. and L. Knaack, "Evaluating the learning in learning objects," Open Learning, vol. 22, no. 1, 2007, pp. 5-28.

[10] X. Ochoa and E. Duval, "Relevance ranking metrics for learning objects," IEEE Trans. Learn. Technol., vol. 1, January 2008, pp. 34-48.

[11] J. Sanz-Rodriguez, J. M. Dodero, and S. Sanchez-Alonso, "Metrics-based evaluation of learning object reusability," Software Quality Control, vol. 19, March 2011, pp. 121-140. 
[12] J. J. Sanz, J. M. Dodero, and S. Sánchez, "Ascertaining the relevance of open educational resources by integrating various quality indicators," Revista de Universidad y Sociedad del Conocimiento (RUSC), vol. 8, issue 2, 2011, pp. 211-224.

[13] Y. Akpinar, "Validation of a learning object review instrument: relationship between ratings of learning objects and actual learning outcomes," Interdisciplinary Journal of Knowledge and Learning Objects, vol. 4, 2008, pp. 291-302.

[14] R. G. Baraniuk, "Challenges and opportunities for the open education movement: a connexions case study," in Opening Up Education: The Collective Advancement of Education through Open Technology, Open Content, and Open Knowledge, California: MIT Press, 2007 pp.116-132.

[15] S. Cueva and G. Rodríguez, "OERs, Standards and Trends," Revista de Universidad y Sociedad del Conocimiento (RUSC), vol. 7, no 1 . UOC. 2007

[16] S. Fox, K. Karnawatt, and M. Mydland, "Evaluating implicit measures to improve web search," ACM Transactions on Information Systems, vol. 23, no. 2, 2005, pp. 147-168.

[17] R. Daniel, R. Rehak and R. Mason, "Engaging with the Learning Object Economy," in Littlejohn, Allison, Reusing Online Resources: A Sustainable Approach to E-Learning, London: Kogan Page, 2003 pp. 22-30.

[18] F. Juan, M. Cervera, C. Fernández, and S. Sánchez-Alonso, "Quality metrics in learning objects," Metadata and Semantics, 2009, pp. $135-141$.

[19] A. C. Laverde, Y. S. Cifuentes, Rodriguez, and H. Y. Rincon, "Towards an instructional design methodology based on learning objects," Educational Technology Research and Development, vol. 55 , no. 6 , pp. 671-681.
[20] A Preliminary Analysis of Software Engineering Metrics-based Criteria for the Evaluation of Learning Object Reusability. [Online]. Available: http://online-journals.org/i-jet/article/viewArticle/794

[21] R. F. Kenny, B. Andrews, M. V. Vignola, M. A. Schilz, and J. Covert, "Towards guidelines for the design of interactive multimedia instruction: fostering the reflective decision-making of preservice teachers," Journal of Technology and Teacher Education, vol. 7, no. 1, 1999, pp. 13-31.

[22] K. Han, "Quality rating of learning objects using bayesian belief networks," PhD thesis, Canada: Simon Fraser University. 2004

[23] Ochoa and Duval. (2008). Learnometrics: metrics for learning objects. [Online].

Available: https://lirias.kuleuven.be/bitstream/1979/1891/2/ThesisFinal.pdf

[24] G. Brownfield and R. Oliver, "Factors influencing the discovery and reusability of digital resources for teaching and learning," in Interact, Integrate, Impact: Proc. of the 20th Annual Conference of the Australasian Society for Computers in Learning in Tertiary Education, G. Crisp, D. Thiele, I. Scholten et al. (eds.), Adelaide, 2003, pp. 74-83.

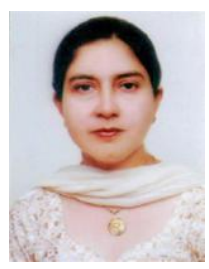

Sonal Chawla is an associate professor at the Department of Computer Science and Applications, Panjab University, India.. She is currently working on Semantic Web Applications, Cloud Computing and Adaptive Education. She has a number of National and International Publications in reputed journals. Besides being on the editorial board she has also been a reviewer and session chair at many National and International Conferences. She has been conferred Career Award for Young Teachers (CAYT) by the All India Council for Technical Education (AICTE), India. 